\title{
Immunohistochemical and molecular features of gastric hyperplastic polyps
}

\author{
Abstract \\ Context: Gastric hyperplastic polyps are generally considered to be inflammatory \\ lesions that develop in association with gastritis. Large polyps may contain foci \\ of dysplasia, suggesting that they represent neoplasms, although molecular data \\ regarding their pathogenesis are lacking.
}

Objective: To describe the clinical, pathologic, and molecular features of large $(>1 \mathrm{~cm})$ gastric hyperplastic polyps and compare them to those of small $(\leq 1 \mathrm{~cm})$ hyperplastic polyps and gastric adenomas.

Design: 15 large hyperplastic polyps $(>1 \mathrm{~cm})$, including 4 with dysplastic foci, 10 small hyperplastic polyps $(\leq 1 \mathrm{~cm})$, and 9 gastric adenomas were evaluated for immunohistochemical expression of MGMT, MLH-1, $\beta$-catenin, p27, p16, and p53. Polyps with dysplasia or abnormal $\beta$-catenin expression were assessed for APC and $\beta$-catenin (CTNNB1) mutations. Large hyperplastic polyps and adenomas were analyzed for KRAS and BRAF mutations.

Results: Complete loss of MGMT expression was significantly more frequent in large hyperplastic polyps $(47 \%)$, often in combination with loss of p27 (47\%), compared to adenomas $(0 \%$ and $0 \%, \mathrm{p}=0.02$, respectively) and smaller lesions $[0 \%(\mathrm{p}=0.02)$ and $10 \%(\mathrm{p}=0.09)$, respectively]. All non-dysplastic hyperplastic polyps showed preserved MLH1 and lacked nuclear $\beta$-catenin staining, but 3 with dysplasia had APC or $\beta$-catenin mutations. KRAS or BRAF mutations were detected in $20 \%$ of large hyperplastic polyps and $11 \%$ of adenomas. Most polyps were entirely negative for $\mathrm{p} 16$, although 6 (40\%) large hyperplastic polyps and $3(33 \%)$ adenomas showed expression in $<10 \%$ of the epithelium. None of the polyps showed $>10 \%$ p 53 expression in the epithelium.

Conclusion: Small gastric hyperplastic polyps are likely non-neoplastic, reparative lesions, but large polyps often show immunohistochemical and molecular abnormalities such as concomitant loss of MGMT with p27 staining, APC or $\beta$-catenin mutations in areas of dysplasia, and KRAS or BRAF mutations. These findings raise the possibility that some large hyperplastic polyps of the gastric mucosa represent early neoplasms.

Keywords: stomach, serrated polyp, adenoma, dysplasia, mgmt, $\beta$ catenin, microsatellite instability, KRAS, BRAF

\author{
Volume 2 Issue I - 2017
}

\author{
Jad Saab, Jeannelyn S Estrella, Yao Tseng Chen, \\ Rhonda K Yantiss \\ Department of Pathology and Laboratory Medicine, Cornell \\ University, USA
}

\begin{abstract}
Correspondence: Rhonda K Yantiss, Department of Pathology and Laboratory Medicine,Weill Cornell Medicine, 525 East 68th Street, New York, NY 10065, USA, Fax 21 2-746-8624, Tel 21 2-
\end{abstract}

746-2824, Email rhy200I@med.cornell.edu

Received: October 30, 2016 | Published: February 09, 2017

\section{Introduction}

Gastric hyperplastic polyps are common epithelial polyps of the stomach encountered in $0.4-1.0 \%$ of upper endoscopic examinations. ${ }^{1,2}$ Together with fundic gland polyps, these lesions account for approximately $80 \%$ of all gastric polyps. ${ }^{1}$ Most gastric hyperplastic polyps show regenerative epithelial changes, characterized by architecturally distorted, cystically dilated glands lined by foveolartype epithelial cells, elongated pits and edematous inflamed lamina propria. Lesional epithelial cells may contain voluminous mucinous cytoplasm or show mucin depletion, but cytologic atypia is minimal or mild. ${ }^{3}$ Foci of intestinal metaplasia are present in about $16 \%$ of cases. Most gastric hyperplastic polyps are solitary sessile lesions that span $<1 \mathrm{~cm}$. They show a predilection for the antrum, although polyps also occur in the cardia near the gastro esophageal junction, in the body, or at gastroenteric anastomoses. Twenty percent of affected patients have multiple polyps, especially those with autoimmune gastritis. ${ }^{1,4}$ These lesions have been traditionally considered to be post-inflammatory regenerative polyps due to their frequent association with mucosal inflammation: $85 \%$ are associated with some type of gastric injury, including autoimmune gastritis, Helicobacter pylori (H. pylori) infection, prior surgical anastomosis, and chemical gastropathy. ${ }^{4}$
Approximately one-third of hyperplastic polyps measure $1 \mathrm{~cm}$ or more in diameter, and some contain foci of dysplasia or carcinoma. ${ }^{4}$ The occurrence of dysplasia in these large lesions is difficult to reconcile with the concept of a post-inflammatory/reparative polyp and raises the possibility that some large gastric hyperplastic polyps are neoplasms, similar to large serrated polyps of the colon, previously considered to be hyperplastic polyps. ${ }^{3-8}$ However, in contrast to colorectal hyperplastic polyps, which have been extensively studied and shown to harbor frequent $B R A F$ or KRAS mutations, the immunohistochemical and molecular features of gastric hyperplastic polyps have not been systematically evaluated, and their relationship to dysplastic gastric polyps (adenomas) is largely unknown..$^{9-11}$ Therefore, the aim of this study is to describe the clinical, pathologic, and molecular features of large $(>1 \mathrm{~cm})$ gastric hyperplastic polyps and compare them to those of small $(\leq 1 \mathrm{~cm})$ hyperplastic polyps and gastric adenomas. We utilized a panel of immunohistochemical markers $\left[\mathrm{O}^{6}\right.$-methylguanine DNA methyltransferase (MGMT), MLH1, p27, p16, p53, and $\beta$ catenin] and performed molecular analyses to assess these lesions for alterations affecting the $W n t$ signaling and mitogen activated protein (MAP) kinase (KRAS and $B R A F)$ pathways, in order to gain insight into their pathogenesis. 


\section{Materials and Methods}

\section{Clinical features}

The files of the Department of Pathology and Laboratory Medicine were searched for gastric hyperplastic polyps and gastric adenomas. Exclusion criteria included an underlying polyposis disorder (PTENhamartoma tumor syndrome, juvenile polyposis, familial adenomatous polyposis, and Cronkhite-Canada syndrome) and Menetrier disease. We selected 34gastric polyps for evaluation, including a study group of 15 hyperplastic polyps that measured at least $1 \mathrm{~cm}$ in diameter and 19controls (10 hyperplastic polyps measuring less than $1 \mathrm{~cm}$ and nine adenomas). Information regarding patient age, gender, polyp size and location, prior or concomitant chronic gastritis and/or H. pylori infection, autoimmune gastritis, chemical gastropathy, and prior gastric surgical resection, was obtained from the patients' medical records, endoscopy reports, and pathology reports. Permission for the study was obtained from the institutional review board at our institution.

\section{Pathologic features}

We reviewed hematoxylin and eosin-stained slides prepared from routinely processed, formalin-fixed polypectomy specimens in all cases. The polyps were classified according to previously established criteria and evaluated for the presence of intestinal metaplasia and dysplasia. The latter was graded as low or high according to the WHO classification scheme and noted to be intestinal or foveolar type, as previously described. ${ }^{12}$ When available, concomitant and prior biopsy samples of the non-polypoid mucosa were evaluated for the presence of gastritis, $H$. pylori infection, and chemical gastropathy.

\section{Immunohistochemical studies}

Immunohistochemical stains were performed on $5 \mu \mathrm{m}$-thick formalin-fixed ( $10 \%$ buffered formalin), paraffin-embedded tissue sections using standard techniques and the antibodies enumerated in Table 1. Nuclear staining for MGMT, MLH1, MSH2, PMS2, MSH6, $\mathrm{p} 27, \mathrm{p} 53, \mathrm{p} 16$, and $\beta$-catenin was evaluated in each of the polyps, and separately assessed in hyperplastic and dysplastic areas of polyps that contained both elements. A positive result for MGMT and mismatch repair proteins was defined as complete loss of staining in the lesional epithelium. Loss of staining for p 27 in $>10 \%$ of the lesional epithelium was considered a positive result, as reported by others. ${ }^{13}$ The extent of strong nuclear staining for p53 in the superficial epithelium was recorded and nuclear staining for $\beta$-catenin was graded as absent (negative or weak staining) or present (moderate to strong staining). The extent of strong cytoplasmic and nuclear staining for p16 was also noted.

Table I Immunohistochemical reagents and PCR primers used in the evaluation of gastric polyps

\begin{tabular}{|c|c|c|}
\hline \multicolumn{3}{|c|}{ Immunohistochemical stains } \\
\hline Marker & Dilution & Company \\
\hline \multicolumn{3}{|l|}{ DNA repair } \\
\hline Complete loss of MGMT & $1: 25$ & Neomarkers (Fremont, CA) \\
\hline Complete loss of MLH-I & $1: 25$ & BD biosciences (San Jose, CA) \\
\hline Complete loss of $\mathrm{MSH}-2$ & 0.18055556 & Calbiochem (Cambridge, MA) \\
\hline Complete loss of MSH-6 & 0.18055556 & BD biosciences (San Jose, CA) \\
\hline \multicolumn{3}{|l|}{ Cell cycle regulation } \\
\hline Loss Of P27 & $1: 25$ & Biocare medical (Concord, CA) \\
\hline $\begin{array}{l}\text { Increased PI6 (nucleus and } \\
\text { cytoplasm) }\end{array}$ & Prediluted & MTM laboratories (Westborough, MA) \\
\hline \multicolumn{3}{|l|}{ Tumor suppressor function } \\
\hline Nuclear P53 staining & $\mathrm{I}: 200$ & Biogenex (San Ramon, CA) \\
\hline \multicolumn{3}{|l|}{ Wnt signaling } \\
\hline Nuclear $\beta$-catenin staining & $1: 200$ & BD biosciences (San Jose, CA) \\
\hline \multicolumn{3}{|c|}{ Primers used for PCR amplification and sequencing } \\
\hline Gene evaluated & Forward & Reverse \\
\hline$\beta$-catenin (CTNNBI) Exon 3 & ATGGAACCAGACAGAAAAGC & GCTACTTGTTCTTGAGTGAAG \\
\hline APC segment I & CAGACTTATTGTGTAGAAGA & СTCCTGAAGAAAATTCAACA \\
\hline APC segment 2 & AGGGTTCTAGTTTATCTTCA & TCTGCTTGGTGGCATGGTTT \\
\hline APC segment 3 & TAAGTGGCATTATAAGCCCCAGTG & TGTATAAATGGCTCATCGAGGCTC \\
\hline
\end{tabular}


Table Continued.

\section{Immunohistochemical stains}

\begin{tabular}{lll}
\hline Marker & Dilution & Company \\
\hline APC segment 4 & ACTCCAGATGGATTTTCTTG & GGCTGGCTTTTTGCTTTAC \\
KRAS (codons I2 and 13) & CGTCTGCAGTCAACTGGAAT & AGAATGGTCCTGCACCAGTAA \\
BRAF $(\mathrm{V} 600 \mathrm{E})$ & TGCTTGCTCTGATAGGAAAATG & GACTTTCTAGTAACTCAGCAGC
\end{tabular}

\section{Molecular analysis}

Molecular studies were performed on adenomas and hyperplastic polyps spanning $>1 \mathrm{~cm}$. For each case, the non-lesional tissue was manually removed from unstained sections mounted on glass slides and DNA was extracted from the remaining tissue using the QIAamp DNA MiniKit (Qiagen Sciences, Valencia, CA), followed by PCR amplification. Hyperplastic and dysplastic epithelia were separately dissected from polyps with both types of elements. The primer sequences, all designed to amplify PCR amplicons $<400 \mathrm{bp}$ in size, are enumerated in Table 1. Polyps with dysplasia, and those that showed nuclear $\beta$-catenin staining, were subjected to mutational analysis of exon 3 (glycogen synthetase kinase- $3 \beta$ phosphorylation region) of CTNNB1 and the $A P C$ mutation cluster region (exon 15, codons 1260-1596). The latter was analyzed in overlapping PCR segments with four sets of paired primers. Extracted DNA was also analyzed for mutations in selected regions of KRAS (codons 12 and 13) and BRAF (V600E). PCR products were sequenced bi-directionally using the Big Dye Terminator chemistry and Applied Bio systems Automated 3730 DNA analyzer and analyzed with the aid of Mutation Surveyor ${ }^{\mathrm{TM}}$ software (Soft Genetics, State College, PA).

\section{Statistical analysis}

The clinical, immunohistochemical and molecular features of each group were tallied and compared using the Fisher's exact test. A p value $<0.05$ was considered statistically significant.

\section{Results}

\section{Clinical and pathologic features}

The clinical and pathologic features of the study cases and controls are summarized in Table 2. Most patients were adults (mean age: 63years, median: 71, range: 16-91) and females were more commonly affected (male/female ratio: 7/10). Most polyps in the study and control groups occurred in the antrum. All 15 large hyperplastic polyps contained tortuous, cystically dilated glands lined by foveolar-type epithelium and inflamed lamina propria (Figure 1). Four displayed foci of intestinal metaplasia, which were limited to the polyp in three cases and present in background mucosa in one case. Four (27\%) large polyps in the study group contained areas of low-grade dysplasia, one of which also showed high-grade dysplasia (Figure 1). Prior or concomitant biopsy samples of nonpolypoid gastric mucosa were available for review in twelve study polyps $(80 \%)$, including eight without dysplasia and all four with dysplasia. In eight study polyps without dysplasia, the background mucosa showed gastric injury in six $(75 \%)$, including three $(38 \%)$ with $H$. pylori-related chronic gastritis and three (38\%) with chemical gastropathy. Of the four study polyps with dysplasia, the background mucosa showed gastric injury in two (50\%), including one (25\%) with chemical gastropathy. Eight patients with small hyperplastic polyps had prior, or concomitant, gastric mucosal biopsies of non-polypoid mucosa. Of these, three (38\%) displayed chemical gastropathy, two
(25\%) contained intestinal metaplasia, and one (13\%) showed $H$. pylori-negative chronic gastritis. All gastric adenomas displayed lowgrade dysplasia. These patients had chronic gastritis $(89 \%)$ and $H$. pylori infection (22\%) in the non-polypoid mucosa at rates similar to those of the study group, although intestinal metaplasia was more frequent (67\%).

Table 2 Clinical and pathologic features of gastric hyperplastic and adenomatous polyps

\begin{tabular}{lllll}
\hline $\begin{array}{l}\text { Feature } \\
\text { evaluated }\end{array}$ & $\begin{array}{l}\text { Large } \\
\text { hyperplastic } \\
\text { polyps } \\
\text { without } \\
\text { dysplasia }\end{array}$ & $\begin{array}{l}\text { Large } \\
\text { hyperplastic } \\
\text { polyps with } \\
\text { dysplasia }\end{array}$ & $\begin{array}{l}\text { Small } \\
\text { hyperplastic } \\
\text { polyps }\end{array}$ & Adenomas \\
\hline $\begin{array}{l}\text { Mean Age } \\
\text { (years) }\end{array}$ & 66 & 62 & 59 & 64 \\
$\begin{array}{l}\text { Male/Female } \\
\text { ratio }\end{array}$ & $7-$ Apr & $2-\mathrm{Feb}$ & $6-\mathrm{Apr}$ & $5-\mathrm{Apr}$ \\
$\begin{array}{l}\text { Polyp location } \\
\text { Cardia }\end{array}$ & $3 / 11(27 \%)$ & $0 / 4(0 \%)$ & $1 / 10(10 \%)$ & $0 / 9(0 \%)$ \\
$\begin{array}{l}\text { Body/Fundus } \\
\text { Antrum }\end{array}$ & $2 / 11(18 \%)$ & $2 / 4(50 \%)$ & $4 / 10(40 \%)$ & $3 / 9(33 \%)$ \\
$\begin{array}{l}\text { Mean size } \\
\text { Chronic }\end{array}$ & $1.5 \mathrm{~cm}(55 \%)$ & $2 / 4(50 \%)$ & $5 / 10(50 \%)$ & $6 / 9(67 \%)$ \\
gastritis & $5 / 7(71 \%)$ & $3.8 \mathrm{~cm}$ & $0.9 \mathrm{~cm}$ & $1.7 \mathrm{~cm}$ \\
$\begin{array}{l}\text { H.pylori } \\
\text { infection }\end{array}$ & $3 / 7(43 \%)$ & $0 / 4(0 \%)$ & $0 / 8(0 \%)$ & $2 / 9(22 \%)$ \\
$\begin{array}{l}\text { Autoimmune } \\
\text { gastritis }\end{array}$ & $0 / 7(0 \%)$ & $0 / 4(0 \%)$ & $0 / 8(0 \%)$ & $1 / 9(11 \%)$ \\
$\begin{array}{l}\text { Chemical } \\
\text { gastropathy }\end{array}$ & $3 / 7(43 \%)$ & $1 / 4(25 \%)$ & $3 / 8(38 \%)$ & $2 / 9(22 \%)$ \\
$\begin{array}{l}\text { Intestinal } \\
\text { metaplasia }\end{array}$ & $3 / 7(43 \%)$ & $1 / 4(25 \%)$ & $2 / 8(25 \%)$ & $6 / 9(67 \%)$ \\
\hline
\end{tabular}

\section{Immunohistochemical and molecular features}

DNA repair mechanisms: The immunohistochemical and molecular features of the study cases and controls are summarized in Table 3. Complete loss of MGMT staining was observed in seven (47\%) large hyperplastic polyps, including two with foci of dysplasia that showed loss of MGMT staining in both non-dysplastic and dysplastic areas (Figure 2A \& 2C). In contrast, all 10 small hyperplastic polyps and nine adenomas showed preserved MGMT staining ( $p=0.02$ versus study polyps). Only one (11\%) gastric adenoma showed complete loss of MLH-1 expression, but all remaining study cases and controls displayed preserved staining for MLH1. 


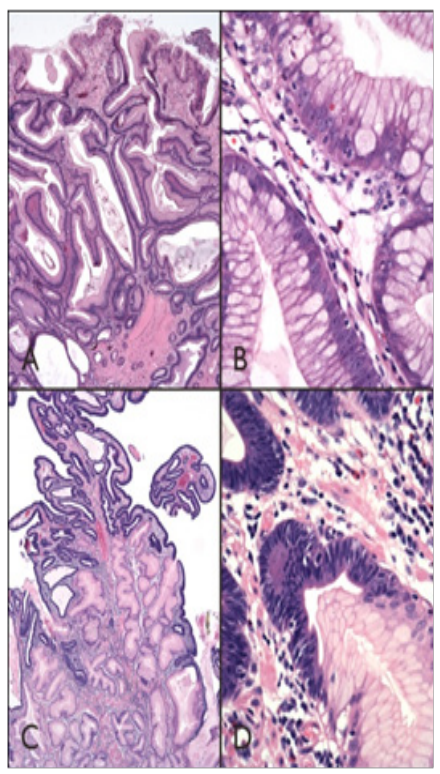

Figure I

(A) Hyperplastic polyps contain tortuous, somewhat dilated glands enmeshed within edematous and inflamed lamina propria.

(B) Epithelial cells may show mucin depletion, or contain abundant cytoplasmic mucin, with mild cytologic atypia.

(C) Four large hyperplastic polyps contained foci of dysplasia.

(D) Which was generally low-grade and displayed nuclear enlargement, hyperchromasia, and increased mitotic activity.
Cell cycle regulation: Seven (47\%) study polyps showed loss of p27 staining, including two with dysplasia that showed decreased expression in both hyperplastic and dysplastic elements (Figure 2D). Five (71\%) study cases with loss of p27 staining showed concomitant loss of MGMT staining. In contrast, p27 staining was preserved in most (90\%) small hyperplastic polyps and all adenomas ( $p=0.09$ and $p=0.02$ versus study polyps, respectively). Nuclear and cytoplasmic p16 staining was observed in $40 \%$ of study polyps, including one with dysplasia and one-third of adenomas, but none $(0 \%)$ of the small hyperplastic polyps (Figure 3A \& 3B). When present, p16 staining was detected in $<10 \%$ of the polyp epithelium and frequently corresponded to moderate or strong nuclear staining for p53 (Figure $3 \mathrm{C} \& 3 \mathrm{D}$ ). None of the study cases or controls showed p53 staining in $>10 \%$ of the lesional epithelium.

Wnt signaling pathway: All large hyperplastic polyps without dysplasia showed membranous $\beta$-catenin staining, whereas nuclear $\beta$-catenin staining was seen in dysplastic areas of two study cases, which also showed mutations in $A P C$ (one case) and $\beta$-catenin (one case) in dysplastic and non-dysplastic areas as well as concomitant loss of MGMT staining (Figure 2B). Another large hyperplastic polyp harbored an $A P C$ mutation limited to areas of dysplasia although nuclear $\beta$-catenin staining was not seen in this case. Five of nine gastric adenomas were successfully analyzed for $A P C$ mutations and all five harbored truncating mutations; $\beta$-catenin mutations were not detected in any of these polyps.

Mitogen activated protein (MAP) kinase pathway: Two study cases showed KRAS mutations, including one with a mutation confined to a dysplastic area, and one large hyperplastic polyp contained a $B R A F$ mutation. Only one $(11 \%)$ gastric adenoma contained a $B R A F$ mutation; all of the rest showed no abnormalities in BRAF or KRAS.

Table 3 Immunohistochemical and molecular features of hyperplastic and dysplastic polyps

\begin{tabular}{|c|c|c|c|c|c|}
\hline & \multirow{2}{*}{$\begin{array}{l}\text { Large } \\
\text { hyperplastic } \\
\text { polyps } \\
\text { without } \\
\text { dysplasia }\end{array}$} & \multicolumn{2}{|c|}{ Large hyperplastic polyps with dysplasia } & \multirow{2}{*}{$\begin{array}{l}\text { Small } \\
\text { hyperplastic } \\
\text { polyps }\end{array}$} & \multirow{2}{*}{ Adenomas } \\
\hline & & Hyperplastic areas & Dysplastic areas & & \\
\hline \multicolumn{6}{|c|}{ DNA repair mechanisms } \\
\hline Loss of MGMT & $5 / I I(45 \%)$ & $2 / 4(50 \%)$ & $2 / 4(50 \%)$ & $0(0 \%)^{*}$ & $0(0 \%) *$ \\
\hline Loss of MLH-I & $0(0 \%)$ & $0(0 \%)$ & $0(0 \%)$ & $0(0 \%)$ & $1 / 9(11 \%)$ \\
\hline Loss of MSH-2 & $0(0 \%)$ & $0(0 \%)$ & $0(0 \%)$ & $0(0 \%)$ & $0(0 \%)$ \\
\hline Loss of MSH-6 & $0(0 \%)$ & $0(0 \%)$ & $0(0 \%)$ & $0(0 \%)$ & $0(0 \%)$ \\
\hline \multicolumn{6}{|l|}{ Cell cycle regulators } \\
\hline Loss of p 27 & $5 / 1(45 \%)$ & $2 / 4(50 \%)$ & $2 / 4(50 \%)$ & $1 / 10(10 \%)$ & $0(0 \%)^{*}$ \\
\hline Increased p 16 & $5 / 1(45 \%)$ & $0 / 4(0 \%)$ & $\mathrm{I} / 4(25 \%)$ & $0(0 \%)$ & $3 / 9(33 \%)$ \\
\hline \multicolumn{6}{|c|}{ Tumor suppressor function } \\
\hline Increased p53 (>10\%) & $0(0 \%)$ & $0(0 \%)$ & $0(0 \%)$ & $0(0 \%)$ & $0(0 \%)$ \\
\hline \multicolumn{6}{|c|}{ Wnt signaling pathway } \\
\hline \multicolumn{6}{|c|}{ Immunohistochemistry } \\
\hline Nuclear $\beta$-catenin & $0(0 \%)$ & $0(0 \%)$ & $2 / 4(50 \%)$ & $0(0 \%)$ & $4 / 9(44 \%)$ \\
\hline Molecular analysis§ & & $N=3$ & $N=3$ & & $N=5$ \\
\hline APC Mutation & $\mathrm{N} / \mathrm{A}$ & I case & 2 cases & $\mathrm{N} / \mathrm{A}$ & 5 cases \\
\hline$\beta$-catenin mutation & $\mathrm{N} / \mathrm{A}$ & I case & I case & $\mathrm{N} / \mathrm{A}$ & 0 cases \\
\hline
\end{tabular}


Table Continued.

\begin{tabular}{|c|c|c|c|c|c|}
\hline & \multirow{2}{*}{$\begin{array}{l}\text { Large } \\
\text { hyperplastic } \\
\text { polyps } \\
\text { without } \\
\text { dysplasia }\end{array}$} & \multicolumn{2}{|c|}{ Large hyperplastic polyps with dysplasia } & \multirow{2}{*}{$\begin{array}{l}\text { Small } \\
\text { hyperplastic } \\
\text { polyps }\end{array}$} & \multirow{2}{*}{ Adenomas } \\
\hline & & Hyperplastic areas & Dysplastic areas & & \\
\hline$K R A S$ mutation & $1 / 11(9 \%)$ & $0(0 \%)$ & $1 / 4(25 \%)$ & $\mathrm{N} / \mathrm{A}$ & $0(0 \%)$ \\
\hline$B R A F$ mutation & $1 / 11(9 \%)$ & $0(0 \%)$ & $0(0 \%)$ & $\mathrm{N} / \mathrm{A}$ & $1 / 9(11 \%)$ \\
\hline
\end{tabular}

$\S$ Discrepancies in total numbers are due to failed analyses and/or insufficient material.

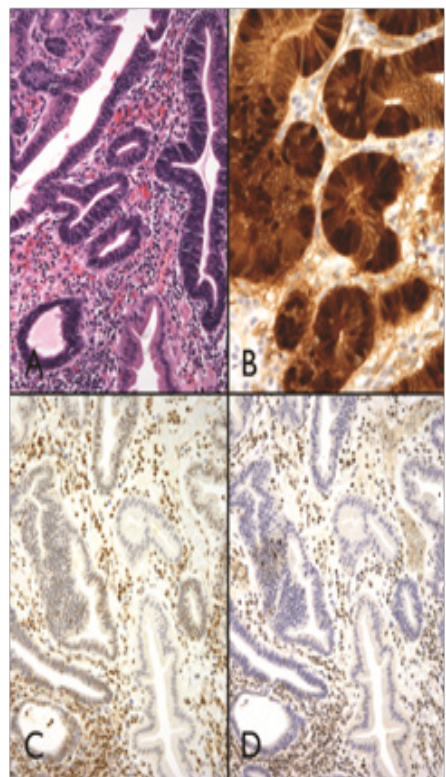

Figure 2: (A) One large hyperplastic polyp with dysplasia.

(B) Showed nuclear $B$-catenin staining that reflected an underlying $B$-catenin mutation.

(C) This lesion also showed concomitant loss of MGMT.

(D) p27 immunohistochemical staining in both non-dysplastic and dysplastic epithelium

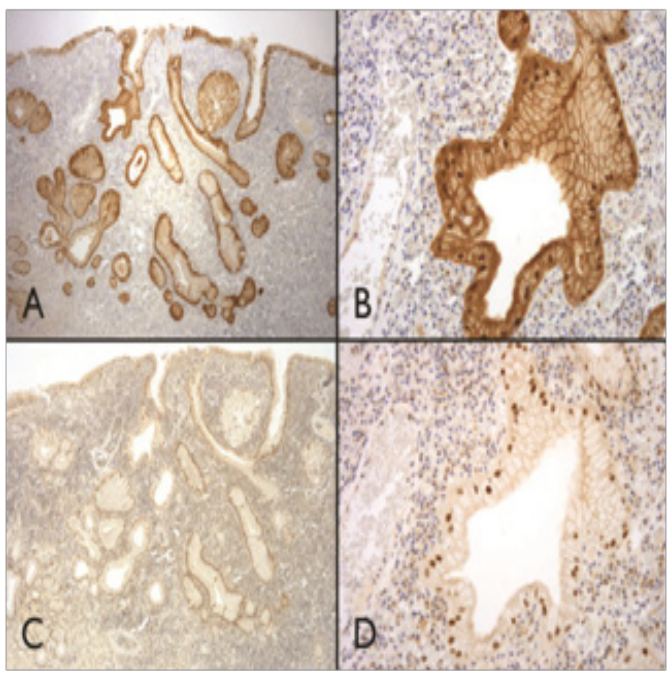

Figure 3

(A \& B) Immunohistochemical studies demonstrated patchy strong nuclear and cytoplasmic pl6 staining.

(C \& D) Which frequently corresponded to moderate or strong nuclear staining for $\mathrm{p} 53$ in virtually every cell

\section{Discussion}

We evaluated the clinical, immuno phenotypic and molecular features of large $(>1 \mathrm{~cm})$ gastric hyperplastic polyps and compared them to the features of small $(\leq 1 \mathrm{~cm})$ hyperplastic polyps and gastric adenomas. We found that large hyperplastic polyps are more frequently associated with chronic gastritis compared to small polyps $(58 \%$ versus $13 \%, p=0.07)$ and show some distinct molecular features. Large hyperplastic polyps commonly display loss of MGMT staining ( $47 \%$ vs. $0 \%$ compared to small polyps, $p=0.02$ ), often in combination with loss of p27 (47\% vs. $10 \%$ compared to small polyps $p=0.09)$, whereas these features were uncommon among gastric adenomas [0\% and $0 \%,(p=0.02)$ for both comparisons]. Large hyperplastic polyps with dysplasia also display abnormalities in the Wnt signaling pathway, as evidenced by either $\beta$-catenin or $A P C$ mutations. At variance with hyperplastic polyps of the colon, KRAS and $B R A F$ mutations are infrequent among hyperplastic and dysplastic polyps of the stomach, suggesting that these mutations are of limited importance in their pathogenesis.

Gastric hyperplastic polyps have been incompletely studied, particularly in comparison to "hyperplastic" polyps of the colorectum. They are generally considered to represent non-neoplastic reparativetype lesions resulting from mucosal inflammation. In a study of 45 patients with gastric hyperplastic polyps, Borch et al. ${ }^{14}$ found $91 \%$ to have underlying chronic gastritis, particularly $H$. pylori-related gastritis $(69 \%)$ [14]. Dirschmid et al. ${ }^{15}$ evaluated the background mucosa of 244 patients with gastric hyperplastic polyps and found $51 \%$ and $37 \%$ to have autoimmune and $H$. pylori-associated gastritis, respectively. ${ }^{15}$ Finally, Abraham et al. ${ }^{4}$ reported that $85 \%$ of hyperplastic polyps developed in combination with chronic gastritis and $37 \%$ occurred in mucosae with intestinal metaplasia. ${ }^{4}$ Our results are similar, although we found small hyperplastic polyps to be more common in cases of chemical gastropathy, whereas large hyperplastic polyps were more frequently associated with chronic gastritis.

Gastric hyperplastic polyps occasionally contain foci of dysplasia. Some describe dysplasia in nearly $20 \%$ of cases, although the true prevalence of dysplasia in these lesions is likely less than 5\%., ${ }^{4,-18}$ Data from several studies including our own suggest that dysplasia is more common in larger hyperplastic polyps. ${ }^{19,20}$ The mechanisms by which dysplasia develops in gastric hyperplastic polyps may be similar to other epithelial neoplasms of the gastrointestinal tract. Hypermethylated promoter regions of mismatch repair genes such as MLH1 and MGMT have been reported in up to $10 \%$ of gastric adenomas, although MSI-H is less frequently identified in $3 \%$ of cases. ${ }^{21-23}$ Only one study to date has examined gastric hyperplastic polyps for mismatch repair deficiency. Nogueira et al. [23] evaluated six hyperplastic polyps for MSI and found only one case with instability at one locus (BAT-26). ${ }^{24}$ We failed to demonstrate evidence for functional mismatch repair deficiency in hyperplastic 
polyps of our study. None of the gastric hyperplastic polyps we examined showed loss of MLH-1 immunostain, although nearly $50 \%$ of large polyps showed complete loss of MGMT expression by immunohistochemistry.

Cyclin dependent kinase inhibitor 1B (CDKN1B, p27) prevents progression into S-phase of the cell cycle and may be important in progression of gastrointestinal neoplasia, particularly in cancers that show extensive DNA hypermethylation. ${ }^{25}$ Gastric adenomas and carcinomas commonly display loss of p27 immunohistochemical staining (up to $30 \%$ and $50 \%$, respectively). ${ }^{26,27}$ In our study, $47 \%$ of large hyperplastic polyps but only $10 \%$ of small hyperplastic polyps, showed loss of p27 staining. Most of these cases (71\%), including one hyperplastic polyp with dysplasia, showed concomitant loss of MGMT staining. Although not conclusive, these findings may point to DNA methylation in the development of large hyperplastic polyps of the stomach.

Abnormalities in the MAP kinase pathway play a minor role in gastric carcinogenesis; less than $5 \%$ of gastric cancers harbor either $K R A S$ or BRAF mutations. ${ }^{28}$ The prevalence of KRAS mutations among gastric adenomas is also low, whereas data regarding mutations among hyperplastic polyps are variable [22,29]. Murakami et al. ${ }^{19}$ found only one KRAS mutation among 17 hyperplastic polyps with dysplasia, but Dijkhuizen et al. ${ }^{29}$ found identical KRAS mutations in three concurrent hyperplastic polyps with high-grade dysplasia from one patient. ${ }^{19,30}$ We found MAP kinase pathway abnormalities in $20 \%$ of large hyperplastic polyps, including two polyps without dysplasia (one having a $B R A F$ mutation and one having a KRAS mutation), and one polyp with dysplasia and a KRAS mutation.

Emerging data suggest that alterations in the Wnt signaling pathway, particularly mutational inactivation of $A P C$, are important to the evolution of gastric epithelial neoplasia. ${ }^{22,29,31}$ Lee et $a .^{22}$ evaluated 78 cases of gastric dysplasia and found $A P C$ mutations in $76 \%$ of cases, but did not detect CTNNB1 mutations in any of the lesions. ${ }^{22}$ Similarly, we detected $W n t$ signaling abnormalities in all gastric adenomas and $67 \%$ of hyperplastic polyps with dysplasia that were tested: two had $A P C$ mutations and one had CTNNB1 mutation. Experience with colorectal neoplasia suggests that alterations in $\beta$-catenin are infrequent compared to $A P C$ mutations, but may be more common among subsets of colon cancer with MSI. Further studies exploring the relationships between MGMT and p27 expression, $\beta$-catenin mutation, DNA methylation, and MSI-H in gastric cancers may elucidate the roles of these factors in gastric carcinogenesis.

Based on our findings and published information, it appears that large gastric hyperplastic polyps are not merely larger versions of small hyperplastic polyps, but biologically different lesions. Features favoring this interpretation include the not infrequent finding of dysplasia in large polyps, their association with inflammation and intestinal metaplasia, and the presence of immuno phenotypic and molecular abnormalities that are more commonly found in neoplastic conditions. Of interest, a study using comparative genomic hybridization showed a similar frequency of chromosomal instability in gastric adenomas and hyperplastic polyps with intraepithelial neoplasia suggesting the possible existence of at least two mechanisms of gastric carcinogenesis, similar to the chromosomal instability and serrated pathways of colorectal carcinogenesis. ${ }^{33}$

\section{Conclusion}

In summary, our results suggest that small gastric hyperplastic polyps are probably non-neoplastic lesions. However, large hyperplastic polyps frequently develop in association with chronic gastritis, show molecular abnormalities, and may even contain foci of dysplasia. Most commonly, large hyperplastic polyps display concomitant loss of MGMT and p27 expression. Abnormalities in $W n t$ signaling, including nuclear $\beta$-catenin immuno expression and/or $A P C$ or $\beta$-catenin mutations, are often detectable in both hyperplastic and dysplastic epithelia of polyps that contain mixed elements. These findings suggest that a subset of large hyperplastic polyps may represent neoplastic lesions that develop secondary to long-standing mucosal inflammatory injury.

\section{Acknowledgements}

None.

\section{Conflict of interest}

The author declares no conflict of interest.

\section{References}

1. Morais DJ, Yamanaka A, Zeitune JM, Gastric polyps: a retrospective analysis of 26,000 digestive endoscopies. Arq Gastroenterol. 2007;44(1):14-17.

2. Carmack SW, Genta RM, Schuler CM, The current spectrum of gastric polyps: a 1-year national study of over 120,000 patients. Am J Gastroenterol. 2009;104(6):1524-1532.

3. Turner Jao, RD. Polyps of the stomach. In: Odze R, Goldblum JR et al. editors. Surgical pathology of the GI tract. liver biliary tree and pancreas, USA: Saunders; 2004. p. 267-271.

4. Abraham SC, Singh VK, Yardley JH, et al. Hyperplastic polyps of the stomach: associations with histologic patterns of gastritis and gastric atrophy. Am J Surg Pathol. 2001;25(10):500-507.

5. Hirano H, Yoshida T, Yoshimura H, et al. Poorly differentiated adenocarcinoma with signet-ring cell carcinoma in a hyperplastic polyp of the stomach: report of a case. Surg Today. 2007;37(10):901-904.

6. Hirasaki S, Suzuki S, Kanzaki H, et al. Minute signet ring cell carcinoma occurring in gastric hyperplastic polyp. World J Gastroenterol. 2007;13(43):5779-5780.

7. Mishra R, Stelow EB, Powell S, et al. Poorly differentiated adenocarcinoma arising from a hyperplastic polyp: which polyps should be endoscopically resected. Dig Dis Sci. 2007;52(6):1586-1588.

8. Orlowska J, Jarosz D, Pachlewski J, et al. Malignant transformation of benign epithelial gastric polyps. Am J Gastroenterol. 1995;90(12):21522159 .

9. Kim KM, Lee EJ, Ha S, et al. Molecular features of colorectal hyperplastic polyps and sessile serrated adenoma/polyps from Korea. Am J Surg Pathol. 2011;35(9):1274-1286.

10. Chan TL, Zhao W, Leung SY, et al. BRAF and KRAS Mutations in Colorectal Hyperplastic Polyps and Serrated Adenomas. Cancer Res. 2003;63(16):4878-4881.

11. Yang S, Farraye FA, Mack C, et al. BRAF and KRAS Mutations in hyperplastic polyps and serrated adenomas of the colorectum: relationship to histology and $\mathrm{CpG}$ island methylation status. $\mathrm{Am} \mathrm{J} \mathrm{Surg} \mathrm{Pa-}$ thol. 2004;28(11):1452-1459.

12. Hamilton SR, Aaltonen LA. World Health Organization Classification of Tumours. Pathology and Genetics of Tumours of the Digestive System, France: IARC Press; 2000

13. Mattioli E, Vogiatzi $\mathrm{P}$, Sun A, et al. Immunohistochemical analysis of pRb2/p130, VEGF, EZH2, p53, p16(INK4A), p27(KIP1), p21(WAF1), Ki-67 expression patterns in gastric cancer. $J$ Cell Physiol. 2007;210(1):183-191. 
14. Borch K, Skarsgard J, Franzen L, et al. Benign gastric polyps: morphological and functional origin. Dig Dis Sci. 2003;48(7):1292-1297.

15. Dirschmid K, Platz Baudin C, Stolte M. Why is the hyperplastic polyp a marker for the precancerous condition of the gastric mucosa?. Virchows Arch. 2006;448(1):80-84.

16. Ginsberg GG, Al Kawas FH, Fleischer DE, et al. Gastric polyps: relationship of size and histology to cancer risk. Am J Gastroenterol. 1996;91(4):714-717.

17. Daibo M, Itabashi M, Hirota T. Malignant transformation of gastric hyperplastic polyps. Am J Gastroenterol. 1987;82(10):1016-1025.

18. Hizawa K, Fuchigami T, Iida M, et al. Possible neoplastic transformation within gastric hyperplastic polyp. Application of endoscopic polypectomy. Surg Endosc. 1995;9(6):714-718.

19. Murakami K, Mitomi H, Yamashita K, et al. p53, but not c-Ki-ras, mutation and down-regulation of p21WAF1/CIP1 and cyclin D1 are associated with malignant transformation in gastric hyperplastic polyps. Am J Clin Pathol. 2001;115(2):224-234.

20. Zea Iriarte WL, Sekine I, Itsuno M, et al. Carcinoma in gastric hyperplastic polyps: a phenotypic study. Dig Dis Sci. 1996;41(2):377-386.

21. Kitajima Y, Miyazaki K, Matsukura S, et al. Loss of expression of DNA repair enzymes MGMT, hMLH1, and hMSH2 during tumor progression in gastric cancer. Gastric Cancer. 2003;6(2):86-95.

22. Lee JH, Abraham SC, Kim HS, et al. Inverse relationship between APC gene mutation in gastric adenomas and development of adenocarcinoma. Am J Pathol. 2002;161(2):611-618.

23. Nogueira AM, Carneiro F, Seruca R, et al. Microsatellite instability in hyperplastic and adenomatous polyps of the stomach. Cancer. 1999;86(9):1649-1656.
24. Kang GH, Lee S, Kim JS, et al. Profile of aberrant CpG island methylation along the multistep pathway of gastric carcinogenesis. Lab Invest. 2003;83(5):635-641.

25. Ogino S, Kawasaki T, Kirkner GJ, et al. Loss of nuclear p27 (CDKN1B/ KIP1) in colorectal cancer is correlated with microsatellite instability and CIMP. Mod Pathol. 2007;20(1):15-22.

26. Yasui W, Kudo Y, Semba S, et al. Reduced expression of cyclin-dependent kinase inhibitor p27Kip1 is associated with advanced stage and invasiveness of gastric carcinomas. Jpn J Cancer Res. 1997;88(7):625629.

27. Oya M, Yao T, Tsuneyoshi M. Expressions of cell-cycle regulatory gene products in conventional gastric adenomas: possible immunohistochemical markers of malignant transformation. Hum Pathol. 2000;31(3):279287.

28. Wu M, Semba S, Oue N, et al. BRAF/K-ras mutation, microsatellite instability, and promoter hypermethylation of hMLH1/MGMT in human gastric carcinomas. Gastric Cancer. 2004;7(4):246-253.

29. Dijkhuizen SM, Entius MM, Clement MJ, et al. Multiple hyperplastic polyps in the stomach: evidence for clonality and neoplastic potential. Gastroenterology. 1997;112(2):561-566.

30. Abraham SC, Park SJ, Lee JH, et al. Genetic alterations in gastric adenomas of intestinal and foveolar phenotypes. Mod Pathol. 2003;16(8):786795.

31. Weiss MM, Kuipers EJ, Postma C, et al. Genome wide array comparative genomic hybridization analysis of premalignant lesions of the stomach. Mol Pathol. 2003;56(5):292-298.

32. Tamura G, Maesawa C, Suzuki Y, et al. Mutations of the APC gene occur during early stages of gastric adenoma development. Cancer Res. 1994;54(5):1149-1151. 